\title{
Analysis of Multipath Effects on FSO Links ${ }^{*}$
}

\author{
Zdenek Kolka $^{1}$, Viera Biolkova ${ }^{1}$ and Dalibor Biolek ${ }^{2}$ \\ ${ }^{1}$ Department of Radio Electronics, Brno University of Technology, Brno, Czech Republic \\ ${ }^{2}$ Department of Electrical Engineering, University of Defence, Brno, Czech Republic \\ kolka@feec.vutbr.cz, biolkova@feec.vutbr.cz, dalibor.biolek@unob.cz
}

\begin{abstract}
The paper evaluates the effect of multipath propagation on broadband terrestrial free-space optical links. At $10 \mathrm{Gbs}$ the pulse duration is $0.1 \mathrm{~ns}$ and multipath propagation during fog events may affect the channel bandwidth and cause intersymbol interference in the receiver. The paper presents in detail a MonteCarlo simulation method based on the Mie scattering theory. All input parameters are clearly linked with FSO link design parameters. Simulation results are provided for typical continental fog parameters and a typical scenario for municipal links.
\end{abstract}

Index Terms - Free-space optical links, scattering, intersymbol interference, Monte Carlo.

\section{Introduction}

The growing demand for multi-gigabit-class wireless broadband terrestrial communication leads to the utilization of radio frequency (RF) bands above $40 \mathrm{GHz}$ for radio systems and infrared bands for Free-Space Optical (FSO) systems. Many propagation studies have shown that near carrier-grade availability can be obtained by combining FSO links with matched-rate millimeter RF links because phenomena that degrade RF link performance and FSO link performance are (almost) mutually exclusive near the ground [1].

Technology for $1.25 \mathrm{Gbs}$ (Ethernet) FSO municipal systems for paths of several kilometers is now available from a few vendors. The next technology step is characterized by data rates of $10 \mathrm{Gbs}$ and above, which requires careful characterization of the atmospheric communication channel. In fact, the advance in FSO technology follows the advance in fiber technology as the key building components (lasers, drivers, and photodiodes) are the same.

The main atmospheric phenomena influencing optical links are scattering on hydrometeors of fog and atmospheric scintillation [1]-[3]. Short-haul municipal FSO systems are designed with a sufficient link margin to accommodate large attenuating effects of clouds and fog. Therefore the effect of atmospheric scintillation during clear-sky periods is not so significant. For lower data rates the atmospheric channel can be modeled as a channel with slowly-varying attenuation, which can reach $120 \mathrm{~dB} / \mathrm{km}$ in moderate continental fog environments [1], [2].

Besides increased attenuation, scattering due to fog also imposes multiple propagation paths, which causes temporal broadening (dispersion) of transmitted pulses, resulting in intersymbol interference (ISI). The ISI introduces a certain power penalty, i.e. the real BER performance of an FSO link differs from laboratory measurements, where the signal is simply attenuated by grey filters.

The time-domain dispersion has been studied for groundto-air/satellite communication through clouds [4], [5] mainly for military purposes, where the analyzed scenarios expected the utilization of multi-kilowatt pulses. Unfortunately, only few papers deal with an analysis of realistic scenarios for terrestrial FSO systems, where the transmitted power is limited by eye-safety regulation and affordable laser sources.

This paper deals with statistical simulation [6] of scattering on water droplets of fog implemented in MATLAB and with the analysis of results for a realistic terrestrial FSO scenario. Section 2 describes the mathematical model used and the implementation of the Monte-Carlo method. Section 3 analyzes the results for a typical $1 \mathrm{~km}$ municipal link.

\section{Model of FSO Path}

\section{A. Power budget equation}

The mean optical power $P_{m, R X A}$ on the receiving aperture of an FSO terminal is [7]

$$
P_{m, R X A}=P_{m, T X A}-\alpha_{f s}-\alpha_{a t m}[\mathrm{~dB}]
$$

where $P_{m, T X A}$ is the mean optical power on the transmitting aperture. For $2 L_{12} \theta_{T X} \gg D_{R X A}$ the free-space path loss $\alpha_{f s}$ can be expressed as

$$
\alpha_{f s}=20 \log \frac{2 L_{12} \theta_{T X}}{D_{R X A}}-3.7,
$$

where $L_{12}$ is the path length, $D_{R X A}$ is the diameter of receiving aperture, and $\theta_{T X}$ is the half angle of beam divergence. For the Gaussian beam the constant $3.7 \mathrm{~dB}$ stems from the definition of $\theta_{T X}$. Attenuation $\alpha_{\text {atm }}$ represents all losses caused by atmospheric phenomena. It is approximately $0.5 \mathrm{~dB} / \mathrm{km}$ for the standard clear atmosphere and may reach hundreds of $\mathrm{dB} / \mathrm{km}$ during fog events [2].

\section{B. Scattering in fog}

Light propagating through fog is scattered on water droplets. As the droplet diameter is comparable to wavelength,

\footnotetext{
* This work has been supported by the Czech Science Foundation under grant No. P102/11/1376, by the Czech Ministry of Industry and Trade under grant agreement No.FR-TI4/148, by the Technology Agency of the Czech Republic under grant agreement No.TA02030845, by the project CZ.1.07/2.3.00/20.0007 WICOMT of the operational programme Education for competitiveness, and by and the Project for the development of K217 Department, UD Brno. The described research was performed in laboratories supported by the SIX project, registration number CZ.1.05/2.1.00/03.0072, operational programme Research and Development for Innovation.
} 
the process is described by the Mie theory. The type of fog is characterized by particle size distribution $n$ (number of particles per unit volume $\left(\mathrm{cm}^{-3}\right)$ per unit increment of radius $(\mu \mathrm{m})$ ), which is usually approximated by the modified gamma distribution [2]

$$
n(r)=a r^{\alpha} \exp (-b r)
$$

where $r$ is the particle radius, and $a, b$, and $\alpha$ are coefficients, see Table I. Radiation (continental) fog generally appears during the night and at the end of the day, particularly in valleys. Advection (maritime) fog is formed by the movement of wet and warm air masses above the colder maritime or terrestrial surfaces. The table gives typical values. Howeveractual fog parameters may vary significantly [2].

TABLE I Fog model parameters [8].

\begin{tabular}{|l|l|l|l|}
\hline Type & $\boldsymbol{a}$ & $\boldsymbol{b}$ & $\boldsymbol{\alpha}$ \\
\hline Dense advection fog & 0.027 & 0.3 & 3 \\
\hline Moderate advection fog & 0.066 & 0.38 & 3 \\
\hline Dense radiation fog & 2.37 & 1.5 & 6 \\
\hline Moderate radiation fog & 607.5 & 3 & 6 \\
\hline
\end{tabular}

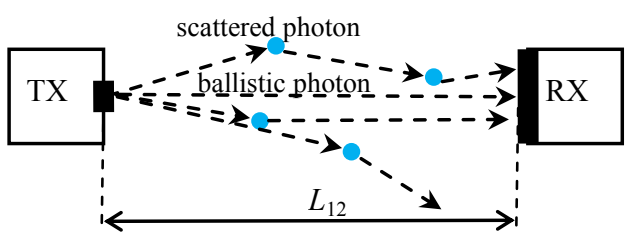

Fig. 1. Atmospheric channel with scattering.

The optical pulse can be regarded as a number of photons that interact with water droplets of fog, Fig. 1. When a photon interacts with a droplet, it is absorbed or randomly deflected from the original direction. The fog density is characterized by the mean distance $d_{a v}$ between two interactions. The optical thickness of fog is then defined as

$$
\tau=L_{12} / d_{a v} .
$$

The distance between two scatterings is an exponential random variable whose $\mathrm{PDF}$ and $\mathrm{CDF}$ are

$$
f_{d}(d)=\exp \left(-d / d_{a v}\right) / d_{a v}, F_{d}(d)=1-\exp \left(-d / d_{a v}\right)
$$

The probability that a "ballistic" photon traveling along the optical axis reaches the receiver without being scattered is

$$
P\left(d>L_{12}\right)=1-F_{d}\left(L_{12}\right)=\exp (-\tau) .
$$

Thus the attenuation due to fog observed by a receiver with narrow field of view will be

$$
\alpha=\tau \operatorname{lolog} e=4.34 \tau[\mathrm{dB}],
$$

which is, in fact, the well-known Beer-Lambert law. During fog events $\alpha_{a t m}$ in (1) is practically given by (7).

When a photon interacts with a droplet, it is absorbed with the probability

$$
P_{a b s}=1-\beta \text {, }
$$

where $\beta$ is the single particle albedo. Otherwise it is scattered at a random angle $\theta$ with arbitrary azimuthal rotation $\varphi$, Fig. 2, [10].

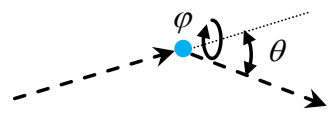

Fig. 2. Single scattering event.

The azimuth angle $\varphi$ is uniformly distributed in $(0,2 \pi)$. The PDF of scattering angle $\theta$ can be obtained from the Mie phase function $P(\theta)$ as

$$
f_{\theta}(\theta)=P(\theta) \sin (\theta) / 2 .
$$

The phase function depends on the wavelength and spectrum of droplet diameters (see Table I). It can be obtained together with the albedo, using, for example, the MiePlot software [11].

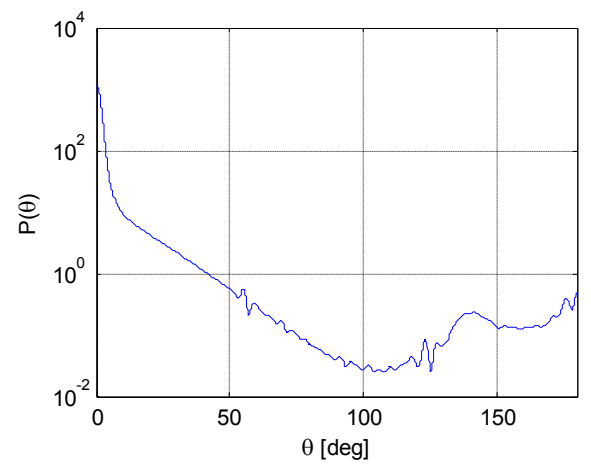

Fig. 3. Mie phase function for dense radiation fog for $\lambda=850 \mathrm{~nm}$.

\section{Geometry of scattering volume}

Let us first examine a single-scattering propagation scenario, which gives a realistic estimation of the time delay between ballistic and scattered photons. Let us assume the scattering volume is constrained conically by the beam divergence half-angle $\theta_{\mathrm{TX}}$ and the receiver field-of-view halfangle $\theta_{\mathrm{RX}}$. Then Fig. 4 shows a situation with the greatest difference between the path lengths.

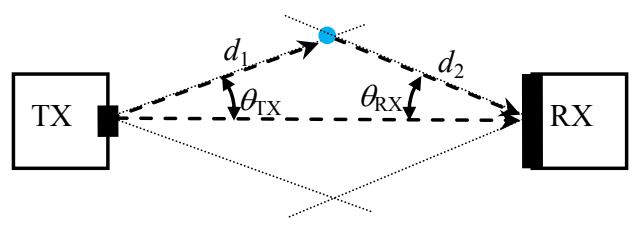

Fig. 4. Single-scattering worst-case scenario. 
The worst-case difference of arrival times $t_{d, \max }$ is

$$
t_{d, \max }=\frac{d_{1}+d_{2}-L_{12}}{c}=\frac{L_{12}}{c}\left(\frac{\sin \theta_{T X}+\sin \theta_{R X}}{\sin \left(\theta_{T X}+\theta_{R X}\right)}-1\right),
$$

where $c$ is the velocity of light. Fig. 5 shows the results for $L_{12}=1 \mathrm{~km}$ and typical values of the half-angles.

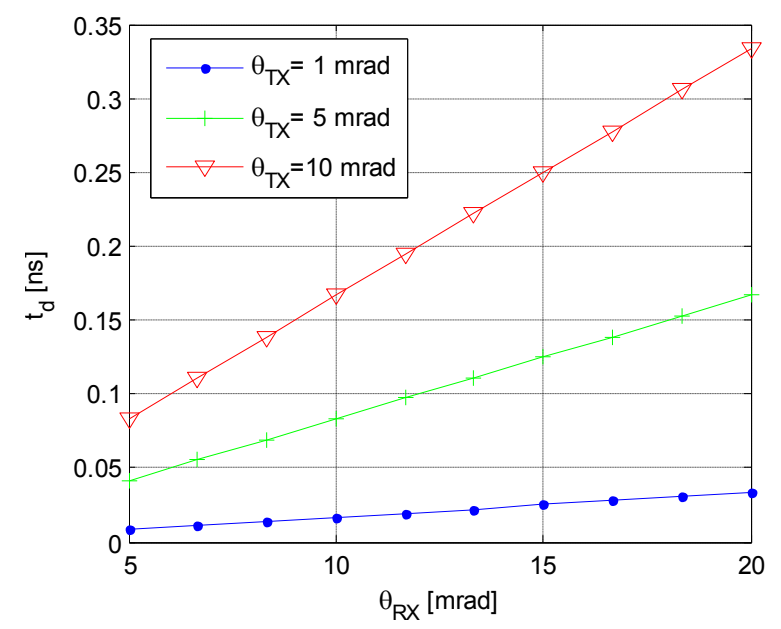

Fig. 5. Worst-cases difference between arrival times of scattered and ballistic photons for single-scattering approximation $\left(L_{12}=1 \mathrm{~km}\right)$.

\section{Monte-Carlo simulation}

As there is no closed model of light propagation in scattering media the results should be obtained via the MonteCarlo analysis, i.e. by tracing each "photon" from a sufficiently large set.

The simulation is based on vector rotations in $3 \mathrm{D}$ space. Let us first present the Rodrigues' rotation formula [12]. Let $\mathbf{v}$ be a vector and $\mathbf{k}$ a unit vector describing an axis of rotation about which we want to rotate $\mathbf{v}$ by an angle $\phi$, then

$\mathbf{v}_{r o t}=R(\mathbf{v}, \mathbf{k}, \phi)=\mathbf{v} \cos \phi+(\mathbf{k} \times \mathbf{v}) \sin \phi+\mathbf{k}(\mathbf{k} \cdot \mathbf{v})(1-\cos \phi)$.

Each photon is represented by its position $\mathbf{p}$ in the $3 \mathrm{D}$ space, the velocity direction vector $\mathbf{v}$, and the normal vector $\mathbf{n}$. Both $\mathbf{v}$ and $\mathbf{n}$ are the unit vectors. The simulation algorithm, which is applied to each photon, is as follows:

1. Generate initial vectors $\mathbf{v}_{0}, \mathbf{p}_{0}$, and $\mathbf{n}_{0}$.

2. Generate random distance $d$ to the next collision using exponential PDF (5a). Update position $\mathbf{p}$ as follows

$$
\mathbf{p}_{i+1}=\mathbf{p}_{i}+d \mathbf{v}_{i} .
$$

3. Delete photons whose position is too far from the beam center from further simulation. Compute exact time of arrival for photons that reach the receiving aperture within the field of view. These photons contribute to the output pulse.

4. Delete photons that will be absorbed, see (8). Generate random rotation $\varphi$ with uniform PDF on $(0,2 \pi)$ and deflection $\theta$ with PDF (9) for remaining photons (see Fig. 2). Modify vectors $\mathbf{v}$ and $\mathbf{n}$ using (11) as follows

$$
\begin{aligned}
& \mathbf{n}_{i+1}=R\left(\mathbf{n}_{i}, \mathbf{v}_{i}, \varphi\right) \\
& \mathbf{v}_{i+1}=R\left(\mathbf{v}_{i}, \mathbf{n}_{i+1}, \theta\right)
\end{aligned}
$$

5. Repeat steps 2 to 4 until no photon remains.

Let the optical beam propagate along the $z$ axis, Fig. 6. To simulate the Gaussian beam the initial deflection $\theta$ from the $z$ axis can be obtained using a random variable with the Rayleigh distribution. The vectors are initialized to

$$
\begin{aligned}
& \mathbf{p}_{0}=(0,0,0)^{T}, \\
& \mathbf{n}_{0}=R\left((1,0,0)^{T},(0,0,1)^{T}, \varphi\right), \\
& \mathbf{v}_{0}=R\left((0,0,1)^{T}, \mathbf{n}_{0}, \theta\right),
\end{aligned}
$$

where random number $\varphi$ has the uniform distribution on $(0,2 \pi)$ and $\theta$ has the Rayleigh distribution whose parameter is $\theta_{\mathrm{TX}} / 2$. Using the actual value of $\theta_{\mathrm{TX}}$ for the initialization leads to the simulation of the free-space path loss (2). For $\theta_{\mathrm{TX}}=0$ the effect is disabled.

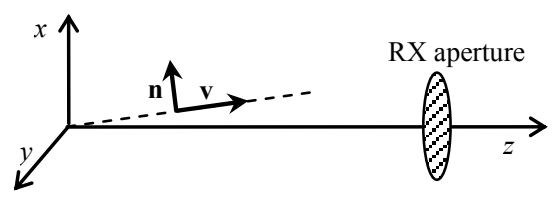

Fig. 6. Coordinate system for Monte-Carlo simulation.

The generation of random numbers with probability density function given in (9) is based on the Smirnov transform [13]. Let $F(x)$ be the cumulative distribution function of (9). Then

$$
\theta=F^{-1}(u)
$$

is a random number whose PDF is (9) provided that $u$ is a uniform random number on $(0,1)$. In practice, $F^{-1}$ can be obtained numerically using a sufficiently dense sampling.

\section{Numerical Study of Typical Municipal Link}

Let us consider a typical municipal FSO system with beam divergence half angle $\theta_{T X}=1.5 \mathrm{mrad}$ and receiving aperture $D_{\mathrm{RXA}}=0.2 \mathrm{~m}$ installed on path $L_{12}=1000 \mathrm{~m}$. The receiver acceptance half angle is $\theta_{R X}=20 \mathrm{mrad}$. Using (2) the freespace path loss is $\alpha_{f s}=19.8 \mathrm{~dB}$. A $10 \mathrm{dBm}$ laser source would give a link margin better than $20 \mathrm{~dB}$ for noise floor $-30 \mathrm{dBm}$.

Figure 7 shows photon positions on the receiving plane $z=L_{12}$ for different number of scattering events $\left(N_{s c}\right)$. The total number of transmitted photons was $10^{5}$. Radiation fog from Fig. 3 was considered with density $\alpha_{a t m}=20 \mathrm{~dB}$ $\left(d_{a v}=217 \mathrm{~m}\right)$. In total 1043 ballistic photons $\left(N_{s c}=0\right)$ was detected on circle given approximately by $2 L_{12} \theta_{\mathrm{TX}}$. The scattered photons, however, can be detected on a much larger area (square $1000 \times 1000 \mathrm{~m}$ shown), i.e. only a small portion hits the receiver aperture. 

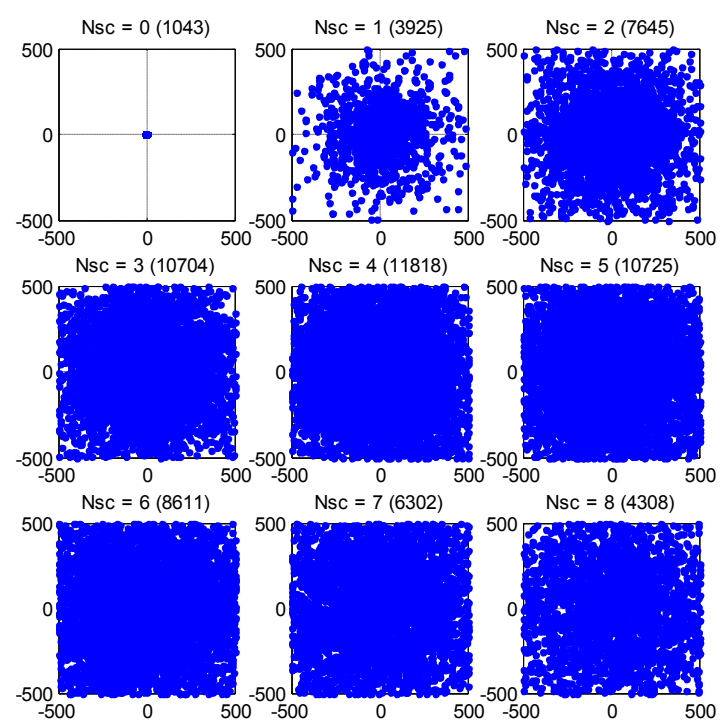

$\mathrm{Nsc}=7(6302)$
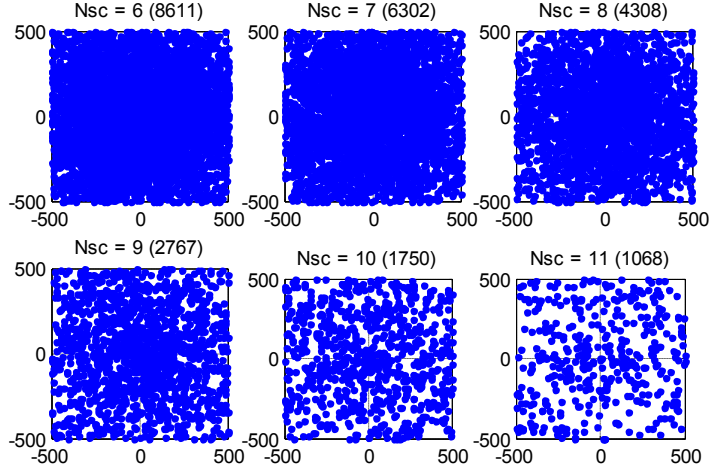

Nsc $=11(1068)$

Fig. 7.Photons at $z=L_{12}$ for different number of scatterings $(1000 \times 1000 \mathrm{~m})$.

The second Monte-Carlo simulation was performed with $10^{7}$ photons for different values of $\alpha_{a t m}$. Table II presents the results. Columns $n_{\text {ball }}$ and $n_{\text {delayed }}$ show the number of received ballistic and delayed photons, respectively.

TABLE II Results of Monte-Carlo simulation.

\begin{tabular}{|c|c|c|c|c|}
\hline$\alpha_{\text {atm }}$ & $\boldsymbol{n}_{\text {ball }}$ & $\boldsymbol{n}_{\text {delayed }}$ & $\boldsymbol{k}_{2}$ & $\boldsymbol{f}_{-3 d B}$ \\
\hline 5 & 27776 & 1682 & $4.5 \cdot 10^{11}$ & $46.1 \mathrm{GHz}$ \\
\hline 10 & 8739 & 1200 & $4.2 \cdot 10^{11}$ & $43.0 \mathrm{GHz}$ \\
\hline 15 & 2826 & 597 & $3.8 \cdot 10^{11}$ & $38.9 \mathrm{GHz}$ \\
\hline 20 & 895 & 270 & $2.5 \cdot 10^{11}$ & $25.6 \mathrm{GHz}$ \\
\hline 25 & 273 & 116 & $2.2 \cdot 10^{11}$ & $22.5 \mathrm{GHz}$ \\
\hline
\end{tabular}

As all photons are "transmitted" at the same time the PDF of arrival times is, in fact, the impulse response of the channel. Several approximations of the delayed pulse have been proposed [6]. The simplest one is the gamma function

$$
h(t)=k_{0} \delta(t)+k_{1} t \exp \left(-k_{2} t\right) \text { for } t \geq 0,
$$

where the Dirac pulse $k_{0} \delta(t)$ represents the ballistic photons arriving at the same time. Coefficients $k_{1}$ and $k_{2}$ can be obtained by curve fitting of the delayed response. The time origin of (19) corresponds to the propagation delay along the line of sight, i.e. $L_{12} / c$. Fig. 8 shows a histogram of arrival times of delayed photons and the approximation. Transforming (19) to the frequency domain it can be found the $3 \mathrm{~dB}$ bandwidth of the delayed response is

$$
f_{-3 d B}=k_{2} \sqrt{\sqrt{2}-1} / 2 \pi
$$

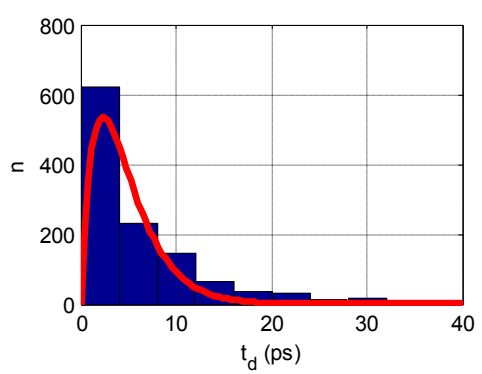

Fig. 8. Histogram of arrival times of delayed photons for $\alpha_{a t m}=10 \mathrm{~dB}$.

\section{Conclusions}

The paper describes in detail an implementation of the Monte-Carlo method for simulation of the multipath propagation of laser pulse in fog. Numerical results for typical municipal link show the channel bandwidth lies in the order of tens of GHz. However, the amplitude of delayed response is lower in comparison with the ballistic photons for low $\alpha_{a t m}$.

\section{References}

[1] F. Nadeem, V. Kvicera, M. S. Awan, E. Leitgeb, S. S. Muhammad, G. Kandus, "Weather Effects on Hybrid FSO/RF Communication Link," IEEE J. Sel. Areas in Comm., vol.27, no.9, Dec. 2009, pp. 1687-1697.

[2] M.S. Awan, E. Leitgeb, S.S. Muhammad, Marzuki, F. Nadeem, M.S. Khan, C. Capsoni, "Distribution Function For Continental and Maritime Fog Environments For Optical Wireless Communication," In Proc. of the 6th Symposium on Communication Systems, Networks and Digital Signal Processing, 2008, Graz, Austria, pp. 260 - 264.

[3] D. Giggenbach, H. Henniger, "Fading-loss assessment in atmospheric freespace optical communication links with on-off keying", Optical Engineering, vol. 47, April 2008, pp. 046001-1 - 046001-6.

[4] E. A. Bucher, R. M. Lerner, "Experiments on Light Pulse Communication and Propagation through Atmospheric Thick Clouds," Appl. Opt. vol. 12, no. 10, pp 2401-2414, 1973.

[5] S. Arnon, D. Sadot, and N. S. Kopeika, "Simple mathematical models for temporal, spatial, angular characteristics of light propagating through the atmosphere for space optical communication: Monte Carlo simulations," J. Mod. Opt., Vol. 41, pp. 1955-1972, 1994.

[6] S. Lee, M. Kavehrad, "Airborne Laser Communications with Impulse Response Shortening and Viterbi decoding," In Proc of IEEE Military Communications Conference (MILCOM 2006), 2006.

[7] S. G. Lambert, W. L. Casey, Communication in Space, London: Artech House, 1995.

[8] D. Harris, "The attenuation of electromagnetic waves due to atmospheric fog", International journal of infrared and millimeter waves, 16(6) 1091-1108 (1995).

[9] J. M. Kahn, W. J. Krause, and J. B. Carruthers, "Experimental characterization of nondirected indoor infrared channels," IEEE Trans. Commun., vol. 43, 1995, pp. 1613-1623.

[10] C. F. Bohren and D. R. Huffman, Absorption and Scattering of Light by Small Particles, John Wiley and Sons, New York, 1983.

[11] http://www.philiplaven.com

[12] G. Taubin, "3D Rotations," IEEE Computer Graphics and Applications, vol.31, no.6, pp.84,89, Nov.-Dec. 2011.

[13] L. Devroye, Non-Uniform Random Variable Generation. SpringerVerlag, New York, 1986. 